05

\title{
Деформация и прочность кварцевых волокон при испытании на 3-точечный изгиб с учетом нелинейной упругости стекла
}

\author{
() Л.Г. Байкова, ${ }^{1}$ Т.И. Песина, ${ }^{1}$ Э.И. Мансырев, ${ }^{2}$ М.Ф. Киреенко, ${ }^{1}$ Л.В. Тихонова ${ }^{1}$ \\ ${ }^{1}$ Физико-технический институт им. А.Ф. Иофрфе РАН, \\ 194021 Санкт-Петербург, Россия \\ ${ }^{2}$ Санкт-Петербургский политехнический университет им. Петра Великого, \\ 195251 Санкт-Петербург, Россия \\ e-mail: Igbaikova@gmail.com
}

(Поступило в Редакцию 18 февраля 2016 г.)

Рассмотрена задача асимметричного распределения деформаций и напряжений в кварцевом волокне при испытании на 3-точечный изгиб. Выполнена оценка параметров нелинейной упругости кварцевого стекла при растяжении и сжатии на основе имеющихся литературных данных. Установлено, что учет нелинейной упругости кварцевого стекла приводит к незначительному увеличению вычисляемых значений прочности по сравнению с данными, полученными на основе линейной теории упругости.

DOI: 10.21883/JTF.2017.01.44016.1764

\section{Введение}

Вопросы прочности и деформации при разрушении кварцевых волокон продолжают привлекать внимание исследователей в связи с широким использованием этих волокон для производства оптических световодов. Современная технология производства оптических кварцевых волокон, используемых в качестве световодов, позволила получить кварцевое волокно в бездефектном состоянии, а нанесение покрытия в ходе производства волокна позволило сохранить поверхность стекла в нетронутом состоянии и обеспечить высокий уровень прочности, присущий структуре стекла [1-3]. Несмотря на то, что оптические кварцевые волокна разрабатываются уже более полувека, вопрос правильного определения их прочности остается актуальным и в настоящее время. В ФТИ исследования структурной прочности стекол проводилось с помощью метода 3-точечного изгиба стеклянных волокон [4,5]. Прочность, как максимальное растягивающее напряжение на поверхности волокна, вычислялась непосредственно по величине разрушающей нагрузки

$$
\sigma=\frac{8 P l}{\pi D^{3}},
$$

где $P$ - разрушающая нагрузка, $l-$ пролет между опорами, $D$ - диаметр волокна $(l=1.02 \mathrm{~mm}, D=125 \mu \mathrm{m})$.

В то же время в ряде работ [6-8] показано, что как при одноосном растяжении, так и при одноосном сжатии, модуль упругости стекла не остается постоянным. Встает вопрос, в какой степени изменение модуля упругости при изгибе волокна может повлиять на определение его прочности при 3-точечном изгибе?

Недавно появилась работа [9], в которой проведен анализ изменения модуля упругости кварцевого стекла с помощью метода бриллюэновского рассеяния света. Работа выполнена на кварцевом волокне с прямоугольным сечением (толщиной $160 \mu \mathrm{m}$, шириной $200 \mu \mathrm{m}$ ).
Было показано, что при 2-точечном изгибе волокна при деформации 6\% происходит смещение нейтральной линии в область растяжения, которая таким образом сужается, а область сжатия увеличивается. Величина модуля упругости при этой деформации возрастает в области растяжения и уменьшается в области сжатия [9].

В связи с этим была поставлена задача провести расчет и анализ максимальных растягивающих напряжений, вызывающих разрушение при 3-точечном изгибе кварцевого волокна, для случая нелинейной зависимости напряжения и модуля упругости от деформации. Это особенно важно при проведении испытаний на прочность в жидком азоте, когда деформации при разрушении могут достигать значений около $18 \%$ [3,10,11]

\section{Законы деформирования кварцевого стекла при растяжении и сжатии}

В общем виде можно описать нелинейную упругость следующим уравнением, ограничиваясь кубической степенью зависимости напряжения $(\sigma)$ от деформации $(\varepsilon)[3]$ :

$$
\sigma(\varepsilon)=Y_{0} \varepsilon+\frac{Y_{1}}{2} \varepsilon^{2}+\frac{Y_{2}}{6} \varepsilon^{3} .
$$

Тогда для модуля упругости $Y(\varepsilon)$, который является производной от напряжения по деформации $(Y \equiv d \sigma / d \varepsilon)$, уравнение будет иметь вид

$$
Y(\varepsilon)=Y_{0}+Y_{1} \varepsilon+\frac{Y_{2}}{2} \varepsilon^{2},
$$

где $Y_{0}$ - модуль Юнга при малых деформациях, $Y_{1}, Y_{2}-$ параметры, определяющие нелинейную зависимость напряжения и модуля упругости от деформации.

Определение этих констант является основной трудностью при обработке результатов, получаемых с помощью методики изгиба. Для этого необходимо на 


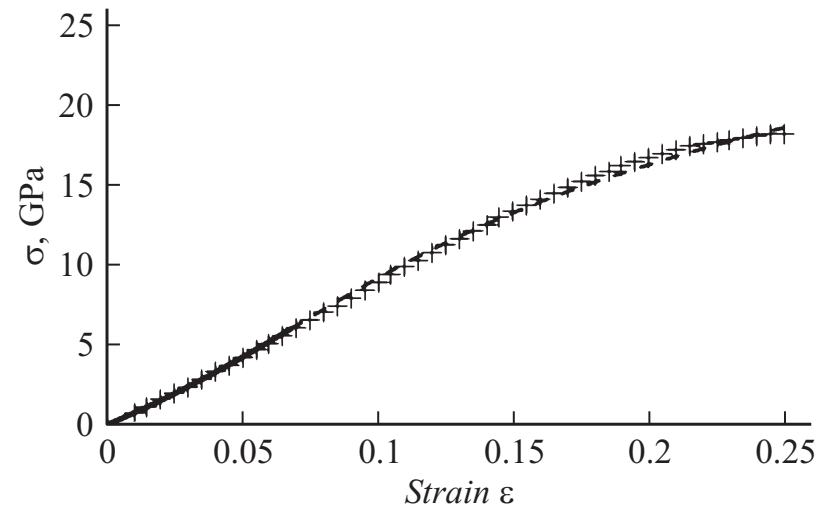

Рис. 1. Зависимость напряжения $(\sigma)$ от деформации $(\varepsilon)$ согласно выражению (4): сплошная линия - по экспериментальным данным [7] до 6\%, штриховая линия - экстраполяция до деформаций $>20 \%,(+)-$ аппроксимация этой зависимости кубическим полиномом $\sigma=Y_{0} \varepsilon+\frac{546.4}{2} \varepsilon^{2}-\frac{6531}{6} \varepsilon^{3}$.

основе имеющихся экспериментальных данных получить зависимость между деформацией и напряжением как для области растяжения, так и для области сжатия.

Нелинейное поведение модуля упругости оптического кварцевого волокна при одноосном растяжении наиболее полно было изучено в работе [7]. В этой работе установлено следующее выражение, связывающее напряжение и деформацию:

$$
\varepsilon=\frac{\sigma}{Y_{0}}-3.2\left(\frac{\sigma}{Y_{0}}\right)^{2}+12\left(\frac{\sigma}{Y_{0}}\right)^{3},
$$

где $Y_{0}=72.3 \mathrm{GPa}$.

К сожалению, экспериментальное исследование зависимости напряжения от деформации было проведено только до деформации 6\%. Эта функциональная зависимость (4) была экстраполирована до деформаций $>20 \%$ и представлена в виде зависимости $\sigma(\varepsilon)$, которая приведена на рис. 1 сплошной линией до деформации $6 \%$ и продолжена штриховой линией в области экстраполяции. Чтобы получить необходимые параметры $Y_{1}$ и $Y_{2}$, зависимость $\sigma(\varepsilon)$ была затем аппроксимирована кубическим полиномом (2). В результате удалось установить следующие коэффициенты, входящие в полином (2): $Y_{1}=546.4, Y_{2}=-6531$. Как видно из рисунка, представление зависимости $\sigma(\varepsilon)$ в области растяжения в виде полинома (2) с найденными параметрами нелинейности $Y_{1}$ и $Y_{2}$ хорошо совпадают с экспериментальной зависимостью $\sigma(\varepsilon)$, полученной в работе [7], и экстраполяцией ее до деформации $\sim 25 \%$.

Следующей задачей являлось определение зависимости модуля упругости от деформации в области сжатия. Поведение модуля упругости кварцевого стекла при одноосном сжатии было изучено в работе [8]. Экспериментально показано, что в области сжатия до деформации
6\% модуль упругости уменьшается согласно уравнению

$$
Y=Y_{0}(1+\alpha|\varepsilon|)
$$

где коэффициент $\alpha=-5.5$. Таким образом, следует, что в выражении (3) $Y_{1}=Y_{0} \alpha$, и при $Y_{0}=72.3 \mathrm{GPa}$ значение параметра $Y_{1}=-398$.

К сожалению, в настоящей работе исследование было проведено в диапазоне деформаций сжатия до 6\%, где наблюдалось только снижение модуля упругости. Из работ по всестороннему сжатию [12] известно, что модуль упругости сначала падает, а потом начинает расти. Поэтому для более полного описания нелинейного поведения модуля упругости в области сжатия при больших деформациях необходимо использовать полином второй степени в зависимости модуля от деформации (см. (3)). Как упомянуто выше, в работе [9] с помощью методики бриллюэновского рассеяния света было проведено исследование упругих модулей при изгибе кварцевого волокна с прямоугольным сечением. Было обнаружено, что в области сжатия модуль Юнга уменьшается до значения $\sim 60 \mathrm{GPa}$. К сожалению, точное положение минимума, после которого модуль упругости должен расти, не было установлено. На основании данных, приведенных в [9], можно предположить, что минимальное значение модуля упругости $\sim 60 \mathrm{GPa}$ находится в области деформаций сжатия $\sim 6.5 \%$. Поскольку производная функции $Y(\varepsilon)$ в минимуме равна нулю, то дифференцируя выражение (3), получим $Y_{1}+Y_{2}|\varepsilon|=0$. Тогда при $Y_{1}=-398$ и $|\varepsilon|=0.065$ значение параметра $Y_{2}=6123$. Таким образом, нелинейное поведение модуля упругости и напряжения в области сжатия можно охарактеризовать коэффициентами $Y_{1}=-398, Y_{2}=6123$.

\section{Закон деформирования при изгибе}

На основании выражения (2) и найденных параметров нелинейности $Y_{1}$ и $Y_{2}$ для областей растяжения и сжатия закон деформирования кварцевого волокна при испыта-

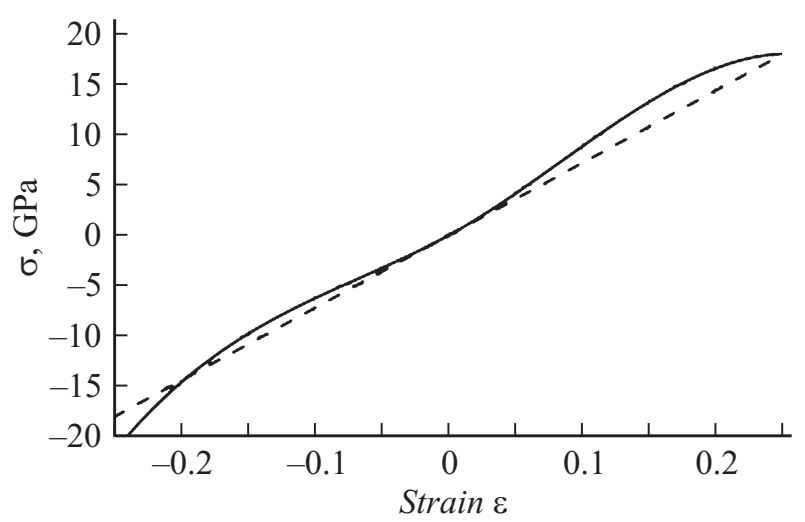

Рис. 2. Диаграмма растяжения-сжатия кварцевого волокна: сплошная линия - согласно закону деформирования (6), штриховая линия - согласно закону Гука. 


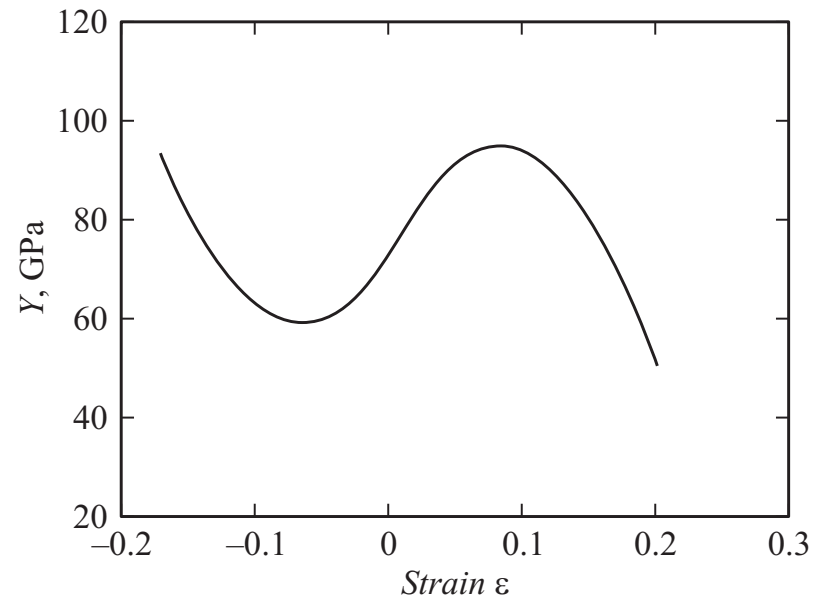

Рис. 3. Зависимость модуля упругости от деформации согласно уравнению (7).

нии на изгиб следует записать в следующем виде:

$$
\sigma(\varepsilon)=\left\{\begin{array}{lll}
Y_{0} \varepsilon+\frac{546.4}{2} \varepsilon^{2}-\frac{6531}{6} \varepsilon^{3} & \text { при } & \varepsilon \geq 0, \\
-\left[Y_{0}|\varepsilon|-\frac{398}{2}(|\varepsilon|)^{2}+\frac{6123}{6}(|\varepsilon|)^{3}\right] & \text { при } & \varepsilon<0 .
\end{array}\right.
$$

Диаграмма растяжения-сжатия для этого закона представлена на рис. 2 (сплошная линия). Для сравнения на этом же рисунке штриховой линией представлена диаграмма растяжения-сжатия для закона Гука: $\sigma=Y_{0} \varepsilon$.

Зависимость модуля упругости от деформации, согласно выражению (3) и найденным параметрам нелинейности, запишется следующим выражением:

$$
Y \varepsilon=\left\{\begin{array}{lll}
Y_{0}+546.4 \varepsilon-\frac{6531}{2} \varepsilon^{2} & \text { при } \quad \varepsilon \geq 0 \\
Y_{0}-398|\varepsilon|+\frac{6123}{2}(|\varepsilon|)^{2} & \text { при } \quad \varepsilon<0 .
\end{array}\right.
$$

Графически она представлена на рис. 3. Во всех выражениях принято $Y_{0}=72.3 \mathrm{GPa}$.

Как следует из закона деформирования (6) и рис. 2, зависимость между напряжениями и деформациями различна при растяжении и сжатии, т. е. задача их нахождения является асимметричной.

В этом случае нейтральный слой в поперечном сечении волокна не будет совпадать со срединным слоем, а сместится в сторону центра кривизны оси волокна. Согласно гипотезе Кирхгофа-Лява [13], распределение деформаций по высоте поперечного сечения будет иметь вид

$$
\varepsilon\left(\varepsilon_{0}, y, \rho\right)=\varepsilon_{0}-\frac{y}{\rho},
$$

где $\varepsilon_{0}$ - деформация в срединном слое, $\rho-$ радиус кривизны нейтрального слоя, $y-$ расстояние от произвольного слоя до срединного в рассматриваемом поперечном сечении.

Для определения деформаций в волокне, а затем и напряжений, необходимо определить положение нейтрального слоя и радиус кривизны нейтрального слоя в поперечном сечении волокна. Они находятся из следующих условий равновесия: 1) сумма внутренних сил упругости в поперечном сечении волокна равна нулю и 2) их момент равен изгибающему моменту. Для этого нужно решить два уравнения статики

$$
\int_{A} \sigma d A=0, \quad \int_{A} \sigma y d A=M_{\text {bend }},
$$

где интегрирование ведется по области поперечного сечения волокна $A$.

Нормальная сила $(N)$ в поперечном сечении как функция радиуса кривизны нейтрального слоя $(\rho)$ и деформации в срединном слое $\left(\varepsilon_{0}\right)$ для круглого сечения выразится формулой

$$
N\left(\rho, \varepsilon_{0}\right)=\int_{-\frac{D}{2}}^{\frac{D}{2}} \sigma\left(\varepsilon\left(\varepsilon_{0}, y, \rho\right)\right) 2 \sqrt{\left(\frac{D}{2}\right)^{2}-y^{2}} d y .
$$

А изгибающий момент $\left(M_{\text {bend }}\right)$ как функция радиуса кривизны нейтрального слоя и деформации в срединном слое, определяется выражением

$$
M_{\text {bend }}\left(\rho, \varepsilon_{0}\right)=-\int_{-\frac{D}{2}}^{\frac{D}{2}} \sigma\left(\varepsilon\left(\varepsilon_{0}, y, \rho\right)\right) y 2 \sqrt{\left(\frac{D}{2}\right)^{2}-y^{2} d y .}
$$

Таким образом, чтобы определить деформации и напряжения для предложенного закона деформирования кварцевого волокна (6), необходимо решить систему двух уравнений (9), используя выражения (10) и (11) применительно к экспериментальным данным, полученным при испытании оптического кварцевого волокна на изгиб.

\section{Результаты расчета деформаций и напряжений при разрушении волокна с учетом нелинейной упругости стекла}

При испытаниях на 3-точечный изгиб фиксировалась разрушающая нагрузка $P$, приложенная в центре пролета $[14,15]$, и разрушающий изгибающий момент определялся по следующей формуле: $M=P l / 4$. В зависимости от условий испытания (на воздухе при температуре $20^{\circ} \mathrm{C}$ и относительной влажности $35 \%$ или в жидком азоте при температуре $-196^{\circ} \mathrm{C}$ ) разрушающая нагрузка и, соответственно, изгибающий момент изменялись в широких пределах. На рис. 4 приведены данные для изгибающего момента при разрушении оптического кварцевого волокна на воздухе и в жидком азоте, которые, как и ранее в работе [15], представлены в виде распределения Вейбулла. Как следует из рис. 4 (кривая 1), при испытании на воздухе наблюдается узкое распределение значений изгибающего момента при разрушении, поэтому расчет проводился только для среднего значения момента 


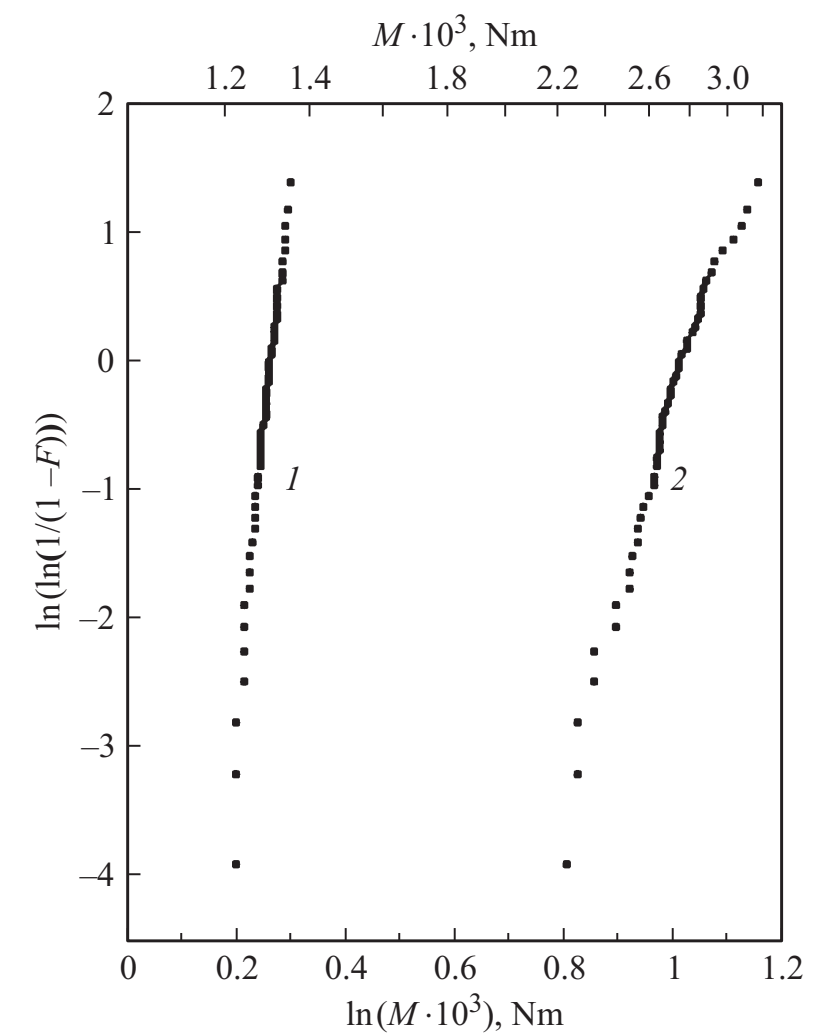

Рис. 4. Вейбулловское распределение изгибающих моментов при испытании на 3-точечный изгиб на воздухе (1) и в жидком азоте (2). Среднее значение $M=1.275 \cdot 10^{-3} \mathrm{Nm}$ при испытании на воздухе и $M=2.678 \cdot 10^{-3} \mathrm{Nm}$ при испытании в жидком азоте (в каждом случае испытано по 50 образцов).

$\left(M=1.275 \cdot 10^{-3} \mathrm{Nm}\right)$. При испытаниях в жидком азоте наблюдалось широкое распределение значений изгибающего момента (рис. 4, кривая 2). В этом случае расчет проводился для среднего значения $M=2.678 \cdot 10^{-3} \mathrm{Nm}$, а также для минимального $M=2.3 \cdot 10^{-3} \mathrm{Nm}$, вычисленного по $10 \%$ нижних значений, и для максимального $M=3.06 \cdot 10^{-3} \mathrm{Nm}$ (по $10 \%$ верхних значений).

Решение поставленной задачи для каждого из этих моментов выполнено с помощью пакета прикладных программ Mathcad 15.0, реализующих численные итерационные методы решения системы нелинейных транс-

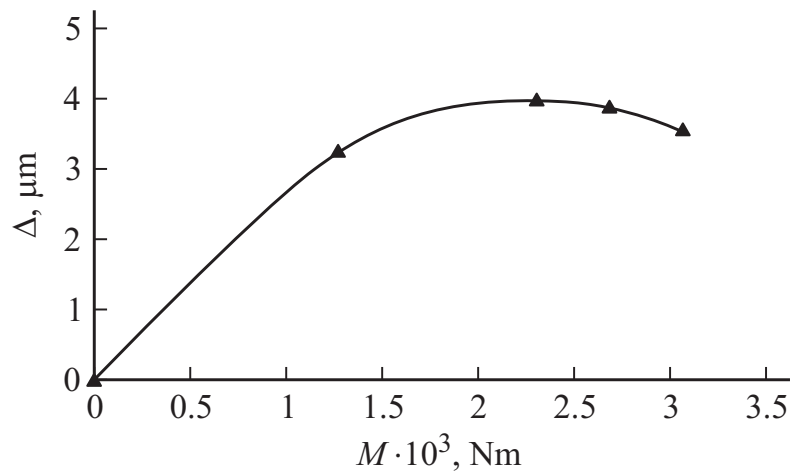

Рис. 5. Зависимость смещения нейтрального слоя $(\Delta)$ от изгибающего момента $(M)$.

цендентных алгебраических уравнений (9) и методы численного интегрирования, реализующие в процессе итераций вычисление интегралов (10) и (11).

Для каждого значения изгибающего момента были определены радиус кривизны нейтрального слоя $(\rho)$ и деформация в срединном слое $\left(\varepsilon_{0}\right)$. Деформация в срединном слое получилась отрицательной, что свидетельствует о смещении нейтрального слоя от срединного в сторону области растяжения в поперечном сечении волокна. Смещение нейтрального слоя вычислялось по формуле $\Delta=\left|\varepsilon_{0}\right| \rho$ и приведено в таблице и на рис. 5.

Вычисленные значения максимальных деформаций и напряжений для каждого изгибающего момента представлены в таблице и на рис. 6 . На рис. $6, a$ представлена зависимость деформации от величины изгибающего момента при 3-точечном изгибе: штриховая линия соответствует расчету деформации при условии $Y_{0}=$ const, сплошная линия проведена через точки, показывающие величину деформаций, вычисленных с учетом нелинейной упругости. Последние на $\sim 10 \%$ ниже, чем значения деформации, полученные на основе линейной теории упругости.

Сравним результаты расчета напряжений, полученные при решении асимметричной задачи, с данными, полученными ранее в работе [5], когда расчет напряжений проводился по формуле (1) без учета нелинейности модуля упругости (см. также таблицу). Графически это представлено на рис. $6, b$. Результаты показаны в ви-

Результаты расчета максимальных деформаций и напряжений в кварцевом волокне при 3-точечном изгибе с учетом нелинейной упругости стекла и при постоянном модуле упругости $Y_{0}$

\begin{tabular}{|c|c|c|c|c|c|c|c|}
\hline \multirow{3}{*}{$\begin{array}{c}\text { Изгибающий } \\
\text { момент } \\
M \cdot 10^{3} \mathrm{Nm}\end{array}$} & \multicolumn{5}{|c|}{ Расчет по уравнениям (10),(11) } & \multirow{2}{*}{\multicolumn{2}{|c|}{$\begin{array}{c}\text { Расчет } \\
\text { по формуле (1) }\end{array}$}} \\
\hline & \multirow{2}{*}{$\begin{array}{c}\text { смещение нейтрального } \\
\text { слоя } \Delta, \mu \mathrm{m}\end{array}$} & \multirow{2}{*}{$\begin{array}{c}\mathcal{E}_{\text {tens }}, \\
\%\end{array}$} & \multirow{2}{*}{$\begin{array}{l}\sigma_{\text {tens }} \\
\mathrm{GPa}\end{array}$} & \multirow{2}{*}{$\begin{array}{c}\varepsilon_{\text {comp }} \\
\%\end{array}$} & \multirow{2}{*}{$\begin{array}{c}\sigma_{\text {comp }}, \\
\mathrm{GPa}\end{array}$} & & \\
\hline & & & & & & $\varepsilon, \%$ & $\sigma, \mathrm{GPa}$ \\
\hline 1.275 & 3.26 & 8.6 & 7.6 & -9.6 & -6.0 & 9.3 & 6.7 \\
\hline 2.300 & 3.96 & 15.0 & 13.4 & -17.1 & -11.6 & 16.6 & 12.0 \\
\hline 2.678 & 3.87 & 17.3 & 15.0 & -19.6 & -14.0 & 19.4 & 14.0 \\
\hline 3.060 & 3.36 & 19.6 & 16.5 & -22.0 & -17.2 & 22.0 & 16.0 \\
\hline
\end{tabular}




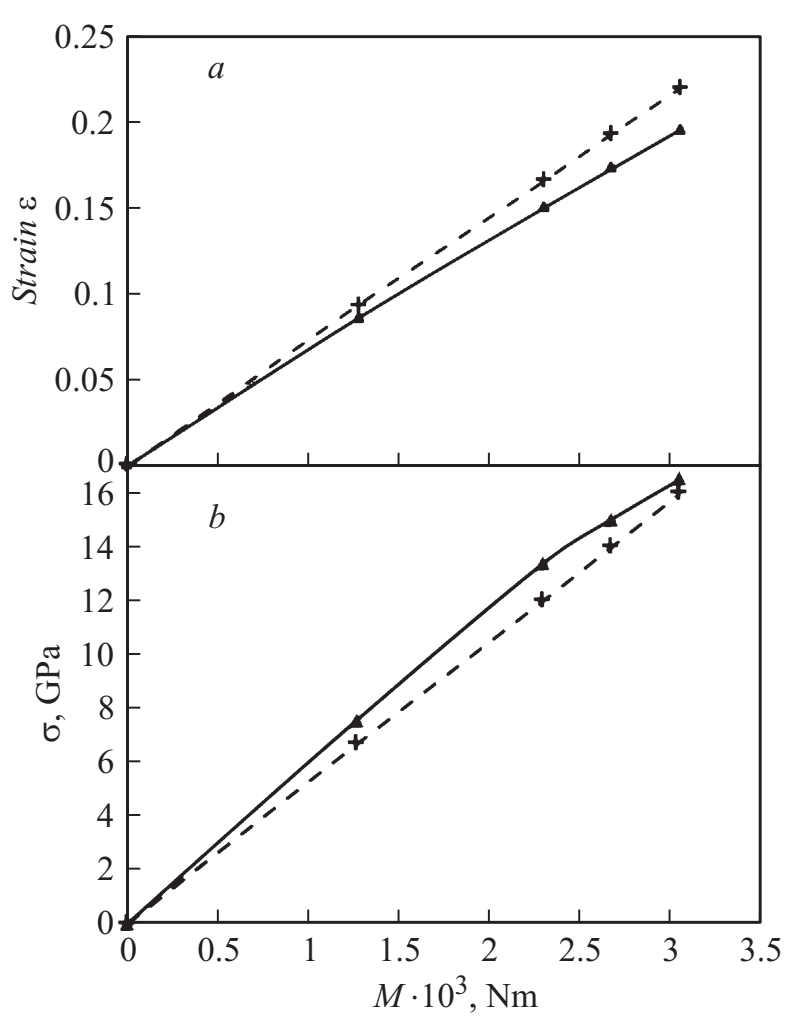

Рис. 6. Зависимость деформации при растяжении (a) и растягивающего напряжения $(b)$ от изгивающего момента. Сплошные линии - согласно закону деформирования (6) и формулам (10),(11), штриховые линии - согласно закону Гука.

де точек, представляющих собой значения прочности, вычисленные для каждого изгибающего момента по формуле (1) для случая линейной упругости (штриховая линия) и по формулам (10), (11) для случая нелинейной упругости (точки соединены сплошной линией). Штриховая линия на рис. $6, b$ демонстрирует прямую пропорциональную зависимость прочности от величины изгибающего момента, как и следует из теории линейной упругости. Сплошная линия демонстрирует более высокие значения прочности, полученные при учете нелинейности упругости. Расхождение между значениями прочности, полученными при решении этих двух задач, не является постоянным и в среднем не превышает $10 \%$.

Из представленных данных можно сделать вывод о том, что расчет по формулам линейной теории упругости дает несколько заниженные значения прочности по сравнению с расчетами на основе уравнений, включающих параметры нелинейного поведения модулей упругости кварцевого стекла при растяжении и сжатии.

Следует отметить, что сделанные количестввенные оценки параметров нелинейной упругости $Y_{1}$ и $Y_{2}$ для кварцевого стекла не являются окончательными, поскольку они базируются на ограниченном экспериментальном материале, представленном в работах [7-9]. Для получения более точных значений параметров нели- нейности необходимы эксперименты по растяжению и сжатию кварцевого стекла при больших деформациях.

\section{Заключение}

Оценки значений прочности оптического кварцевого волокна при испытании на 3-точечный изгиб, полученные прямым расчетом по формулам линейной теории упругости, близки или несколько занижены по сравнению со значениями прочности, вычисленными с учетом нелинейной упругости кварцевого стекла. При отсутствии информации о параметрах нелинейной упругости структурную прочность стекла любого состава можно приближенно с достаточной степенью точности оценивать с помощью методики 3-точечного изгиба по формулам линейной теории упругости.

Авторы выражают свою благодарность Д.С. Рыбину за проведение математической обработки данных работы [7].

\section{Список литературы}

[1] Kurkjian C.R., Paek U.C. // Appl. Phys. Lett. 1983. Vol. 42. N 9. P. 251-253.

[2] Kurkjian C.R., Gupta P.K., Brow R.K., Lower N.P. // J. NonCryst. Sol. 2003. Vol. 316. P. 114-124.

[3] Gupra P.K., Kurkjian C.R. // J. Non-Cryst. Sol. 2005. Vol. 351. P. 2324-2328.

[4] Пух В.П., Песина Т.И., Иванов М.И. // ФХС. 1981. Т. 7. Вып. 3. С. 328-331.

[5] Пух В.П., Байкова Л.Г., Киреенко М.Ф., Тихонова Л.В., Казанникова Т.П., Синани А.Б. // ФТТ. 2005. Т. 47. Вып. 5. C. $850-855$.

[6] Mallinder F.P., Proctor B.A. // Phys. Chem. Glasses. 1964. Vol. 5. P. 91-103.

[7] Krause J.T., Testardi L.R., Thruston R.R. // Phys. Chem. Glasses. 1979. Vol. 20. N 6. P. 135-139.

[8] Griffioen W. // J. Am. Ceram. Soc. 1992. Vol. 75. N 10. P. 2692-2696.

[9] Guerette M., Huang L. // Am. Ceram. Soc. Bull. 2015. Vol. 94. N 4. P. $40-43$.

[10] France P.W., Duncan W.J., Smith D.G., Beales K.J. // J. Mat. Sci. 1983. Vol. 18. P. 785-792.

[11] Kurkjian C.R., Gupta P.K., Brow R.K. // Int. J. Appl. Glass Sci. 2010. Vol. 1. N 1. P. 27-37.

[12] Kondo K., Satoshi Lio, Sawaoka A. // J. Appl. Plys. 1981. Vol. 52. N 4. P. 2826-2831.

[13] Ляв А. Математическая теория упругости. М., Л.: ОНТИ, $1935.674 \mathrm{c}$.

[14] Байкова Л.Г., Песина Т.И., Киреенко М.Ф., Тихонова Л.В., Kurkjian C.R. // ЖТФ. 2015. Т. 85. Вып. 6. C. 83-86.

[15] Байкова Л.Г., Песина Т.И., Kurkjian C.R., Tang Zh., Киреенко М.Ф., Тихонова Л.В., Пух В.П. // ЖТФ. 2013. т. 83. Вып. 10. С. 55-60. 